\title{
Arterialização do arco venoso do pé para tratamento da tromboangeíte obliterante
}

\author{
Arterialization of the venous arch of the foot for the treatment of \\ thromboangiitis obliterans
}

\section{Cesar Roberto Busato ${ }^{1}$, Carlos Alberto Lima Utrabo², Ricardo Zanetti Gomes ${ }^{2}$, Joel Kengi Housome², Eliziane Hoeldtke ${ }^{3}$, Cristiano Teixeira Pinto ${ }^{4}$, Rafael Ignácio Brandão ${ }^{4}$, Cintia Doná Busato ${ }^{5}$}

\section{Resumo}

Em isquemia crítica sem leito arterial distal, um dos modos de irrigar o membro isquêmico é derivar o fluxo de maneira retrógrada através do sistema venoso. As primeiras tentativas de fístulas arteriovenosas terapêuticas datam do início do século passado. Realizadas na parte proximal dos membros inferiores, não obtiveram resultados favoráveis. A partir da década de 70, com os trabalhos pioneiros de Lengua, as fístulas passaram a ser estendidas até o pé, e os bons resultados apareceram em várias publicações. Os autores relatam a evolução de um caso de tromboangeíte obliterante submetida ao procedimento. Essa é uma cirurgia de indicação precisa, que requer estudo pré-operatório arterial e venoso e observância a detalhes de técnica operatória.

Palavras-chave: Arterialização venosa, isquemia crítica, fístulas arteriovenosas terapêuticas, salvamento de membro, tromboangeíte obliterante.

\section{Introdução}

Tentativas de fazer chegar sangue arterial por via venosa retrógrada em territórios de grande isquemia datam do início do século passado com San Martin (1902). Da mesma época são também as constatações de Gallois \& Pinatelle (1903) de que, para vencer os obstáculos das válvulas venosas, faz-se necessária uma força muito maior do que a pressão arterial normal. Helsted

\begin{abstract}
In critical ischemia without arterial run-off, it is possible to irrigate the ischemic limb by turning the course of the flow reversely through the venous system. The first experiments with therapeutic arteriovenous fistulas date from the beginning of the last century. They were performed in the proximal area of the lower limbs, but showed unfavorable results. Since the 1970's, with the pioneer studies of Lengua, fistulas started being extended to the foot and several publications have reported good outcomes. The authors report the evolution of a case of thromboangiitis obliterans which was submitted to the procedure. This is an accurate surgical procedure which requires arterial and venous preoperative study and the observance of technical operative details.
\end{abstract}

Keywords: Venous arterialization, critical ischemia, therapeutic arteriovenous fistulas, limb salvage, thromboangiitis obliterans.

\& Vaughan (1912) criticaram duramente esses procedimentos, embora Roussiel, em 1919, reunindo 63 casos, tenha demonstrado êxito em $25 \%$ deles $^{1}$.

Em 1951, Szilagyi, ícone da cirurgia vascular da época, condenou o método, depois de aplicá-lo em nove casos, que apresentaram $100 \%$ de maus resultados, confeccionando fístulas arteriovenosas em vasos femorais ${ }^{2}$.

Root \& Cruz (1965) e Matolo (1976) demonstraram experimentalmente que as fístulas laterolaterais permitiam um bom retorno venoso e melhores resultados que

1. Professor adjunto, Universidade Estadual de Ponta Grossa, Ponta Grossa, PR. Membro efetivo, SBACV.

2. Membro efetivo, SBACV.

3. Cirurgiã vascular, Santa Casa de Misericórdia de Ponta Grossa, Ponta Grossa, PR.

4. Médico residente, Serviço de Cirurgia Vascular e Angiologia, Santa Casa de Misericórdia de Ponta Grossa, Ponta Grossa, PR.

5 . Acadêmica de Medicina, Faculdade Evangélica de Medicina do Paraná, Curitiba, PR.

O presente estudo foi realizado no Serviço de Cirurgia Vascular Periférica e Angiologia, Santa Casa de Misericórdia de Ponta Grossa, Ponta Grossa, PR.

Apresentado no XXI Conclave Científico de Acadêmicos de Medicina, realizado em Curitiba, PR, em 26 de outubro de 2007.

Não foram declarados conflitos de interesse associados à publicação deste artigo.

Artigo submetido em 14.11.07, aceito em 17.07.08. 
as terminolaterais, cuja sobrecarga venosa levava a edema, equimose e necrose ${ }^{3,4}$.

Abalos, em 1909, foi quem empregou, pela primeira vez, o sistema venoso superficial, para confecção de uma fístula mediante a comunicação da safena magna com a artéria femoral ${ }^{1}$. Courbier, em 1973, e principalmente Lengua com seus trabalhos a partir de 1974, passaram a estender suas fístulas até o pé, obtendo a irrigação dos dedos e melhores resultados do que seus antecessores ${ }^{5}$.

Enxertos venosos com safena reversa derivando fluxo da artéria mais distal e com bom fluxo até o arco venoso do pé foram realizados com sucesso por Porkrowski, Lengua, Chen, Taylor, Engelke, Rowe e Özbek ${ }^{6-17}$. Os autores do presente trabalho, assim como Gasparis, mantêm a safena magna arterializada in situ ${ }^{18,19}$.

Os bons resultados da cirurgia estão relacionados à indicação precisa, estudo pré-operatório arterial e venoso da extremidade em risco e detalhes de técnica operatória. A cirurgia tem indicação precisa para tratamento de isquemia crítica sem leito distal, com a finalidade de tratar a dor em repouso ou promover a cicatrização de amputações menores.

A aterosclerose obliterante, especialmente aquela acompanhada de diabetes melito, a tromboangeíte obliterante na maioria absoluta dos casos e o aneurisma de poplítea com trombose do leito distal são condições em que se encontra com maior freqüência a isquemia crítica sem leito distal. Embora a tromboangeíte obliterante ocorra tanto em veias quanto em artérias, raramente as safenas magna e parva estão acometidas pelo processo inflamatório ${ }^{20}$.

\section{Relato do caso}

Paciente do sexo masculino, com 52 anos, tabagista, com dor em repouso e cianose no pé esquerdo há 30 dias. Refere mancha escura em polpa digital, na mão direita, há 60 dias, que evoluiu com necrose superficial e descamação tecidual. Constatou-se no exame físico pulsos femorais e poplíteos palpáveis e cheios; pedioso direito diminuído; tibiais posteriores e pedioso esquerdo ausentes. Na evolução, apresentou necrose do hálux (Figura 1).

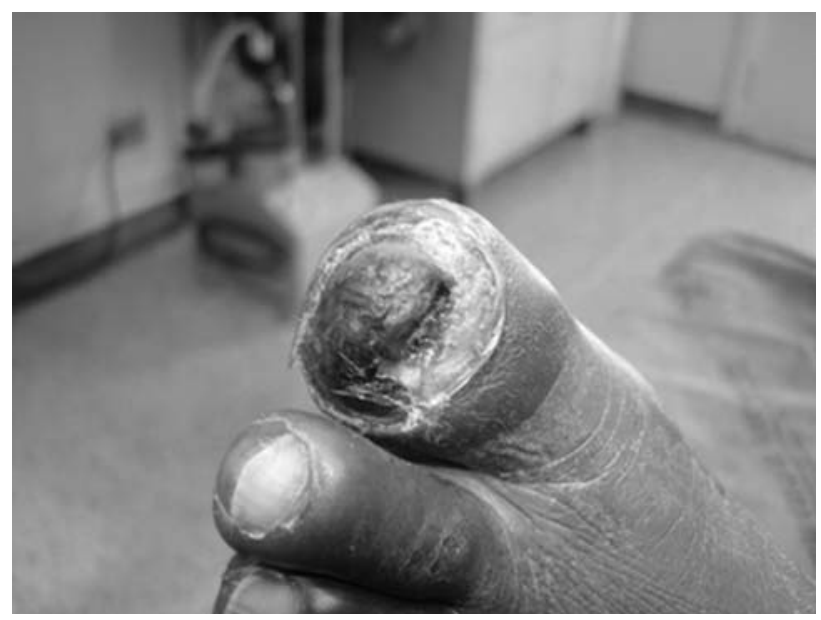

Figura 1 - Necrose do hálux

O mapeamento dúplex e a arteriografia do membro inferior esquerdo (Figura 2) apresentaram artérias femoral e poplítea preservadas, circulação arterial mantida até o terço proximal da perna e ausência de leito distal.

Com diagnóstico de tromboangeíte obliterante, o paciente foi submetido a simpatectomia lombar, apresentando piora da dor. Foi realizada arterialização do arco venoso do pé. A safena magna foi mantida in situ, anastomosada à artéria poplítea (Figura 3) e esqueletizada, pela ligadura das colaterais, até a veia perfurante anterior do maléolo (Figura 4), a partir da qual as veias do pé foram preservadas. Realizamos valvulotomia ao longo da safena, através das colaterais, e no arco venoso

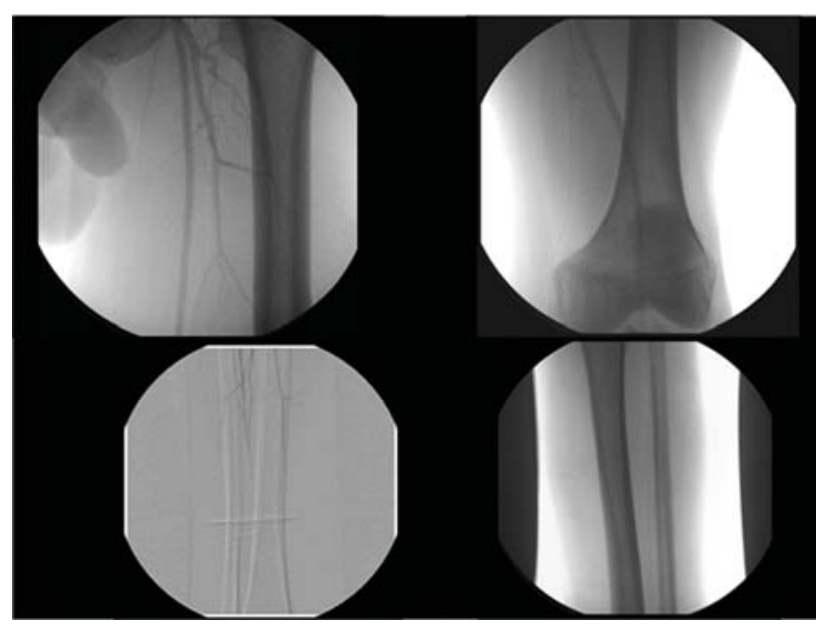

Figura 2 - Arteriografia pré-operatória do membro inferior esquerdo com artérias femoral e poplítea preservadas e artérias distais sem fluxo 


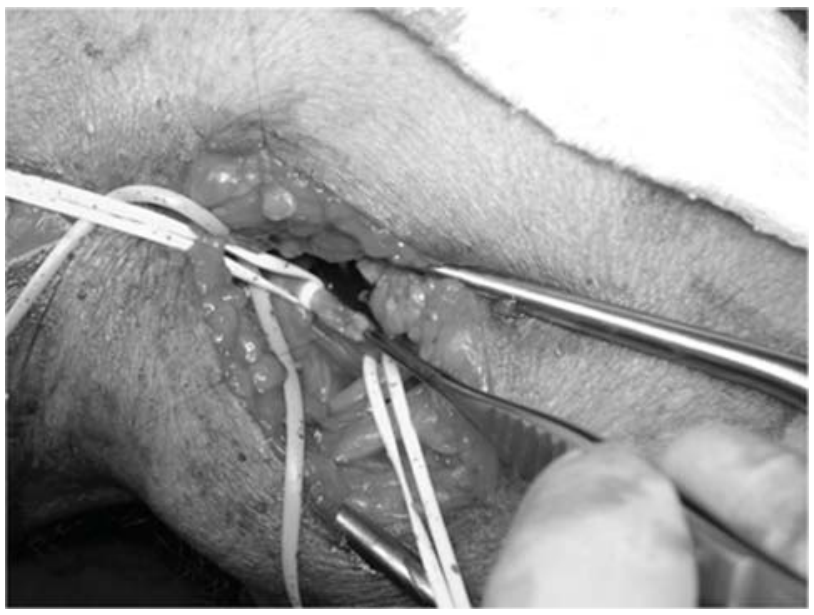

Figura 3 - Anastomose da veia safena magna à artéria poplítea

do pé, via venotomia próxima à emergência das primeiras metatarsianas (para isso, utilizamos o valvulótomo de Mills) (Figura 5). Procedemos à amputação do hálux para cicatrização em primeira intenção.

O paciente evoluiu com aquecimento do membro, melhora da dor, presença de pulso com frêmito no arco venoso do pé e cicatrização do hálux (Figuras 6 a 8).

\section{Discussão}

A arteriografia e o mapeamento dúplex arterial são realizados como rotina à procura de leito para tratamento por enxerto convencional e melhor artéria doadora. O dúplex venoso estuda e marca,

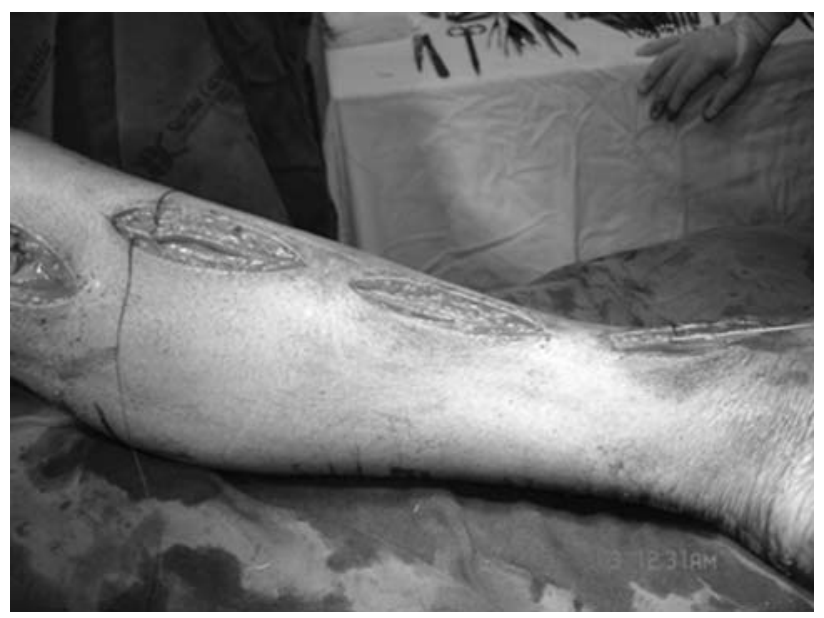

Figura 4 - Ligadura dos ramos da safena magna até a veia perfurante anterior do maléolo (valvulotomia através das colaterais)

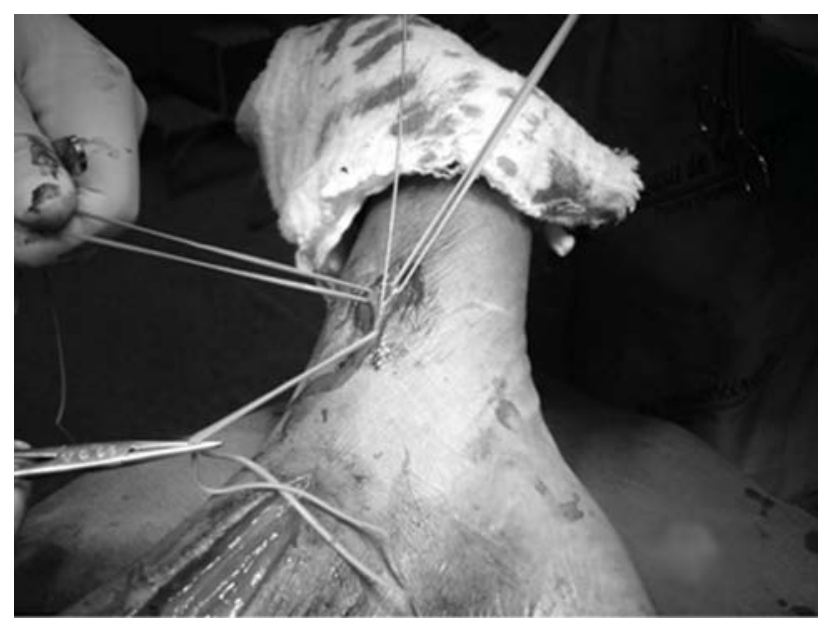

Figura 5 - Dissecção do arco venoso do pé e introdução do valvulótomo através de flebotomia

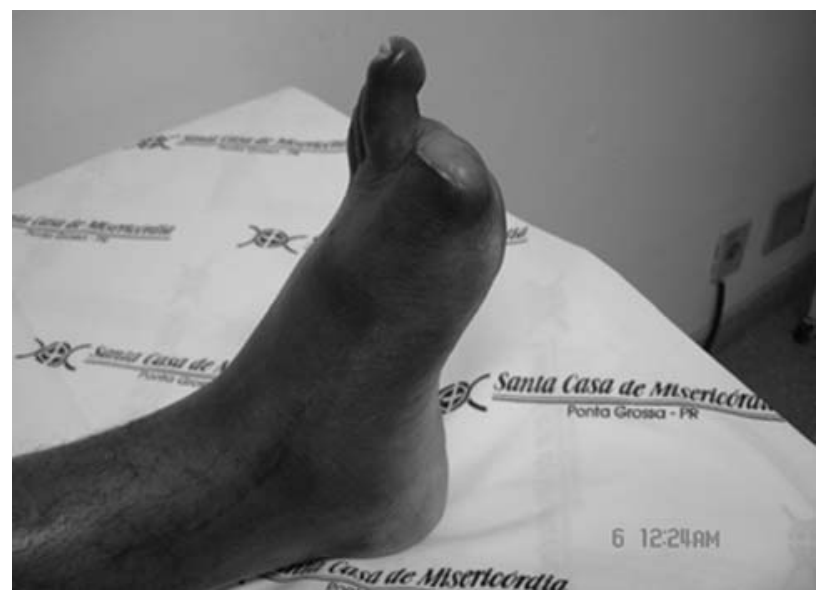

Figura 6 - Hálux após a cirurgia

preferencialmente, a safena magna e sua extensão até o arco venoso do pé, bem como as demais veias do sistema venoso profundo, que garantem o retorno do hiperfluxo gerado pela confecção da fístula.

A técnica cirúrgica pode ser realizada conforme descrita por Lengua, que faz a anastomose distal com safena invertida diretamente no arco venoso ou como preconizamos: mantendo a safena magna in situ ${ }^{12,18}$.

Sherman, em 1949, descreveu oito veias perfurantes na parte medial do pé e sete na lateral. Lofgren, em 1968, injetando, sob pressão, látex em veias perfurantes do arco dorsal entre o primeiro e o segundo metatarsiano, notou enchimento das veias profundas do pé que, por 

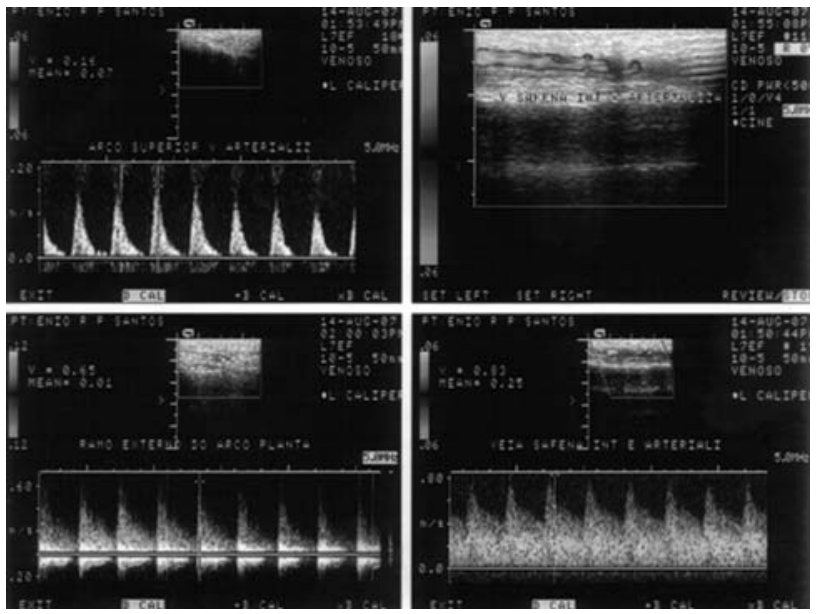

Figura 7 - Dúplex pós-operatório mostrando padrão de fístula arteriovenosa em safena magna e no arco venoso do pé

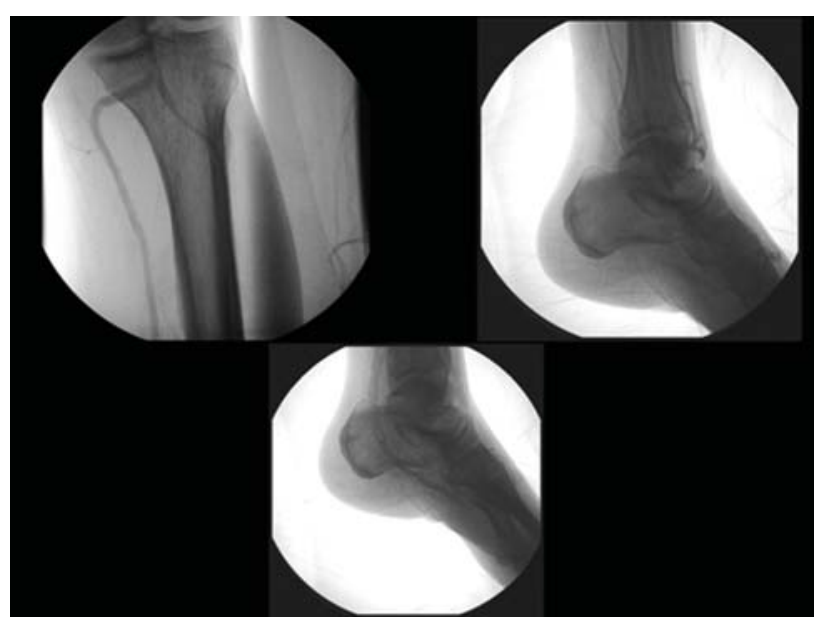

Figura 8 - Arteriografia pós-operatória mostrando anastomose arteriovenosa na poplítea, fluxo arterial pela safena magna no arco venoso do pé e retorno venoso pelo arco plantar

sua vez, encheram as veias superficiais da porção proximal. Dissecando 10 extremidades amputadas, o autor encontrou de 6 a 12 perfurantes, em média 9, que comunicavam o sistema profundo com o superficial. De 94 perfurantes dissecadas, 49 não tinham válvulas, permitindo o fluxo venoso em ambas as direções, 41 tinham uma válvula próxima ao sistema superficial e 4 tinham uma segunda válvula ${ }^{21}$.

Durante a realização de exames flebográficos, os movimentos de flexão dorsal e plantar do pé aspiram contraste, para a circulação profunda. No pé, a maioria das veias perfurantes é desprovida de válvulas, e o fluxo venoso se faz nos dois sentidos. A perfurante de maior importância é a do primeiro espaço interdigital, com aproximadamente $3 \mathrm{~mm}^{22}$. Esses achados podem ser comprovados pela arteriografia pós-operatória do paciente, que apresentou difusão do contraste nos arcos venosos dorsal e plantar.

Em um recente estudo, foram analisadas 56 publicações disponíveis na literatura mundial que utilizaram a arterialização do arco venoso do pé para tratamento de isquemia crítica sem leito distal. Sete trabalhos preencheram os critérios de seguimento, tempo de patência das fístulas e complicações pós-operatórias. Submetidos à metanálise, os estudos perfizeram um total de 228 pacientes com 231 extremidades e um percentual de salvamento de $71 \%$, com cicatrização de lesões, pequenas amputações e melhora da dor em repouso. Do total de casos, 140 eram de aterosclerose e 91 de tromboangeíte obliterante ${ }^{23}$.

Apesar de a literatura mundial recente mostrar um aumento de publicações utilizando o método, um número expressivo de pacientes sem leito distal, especialmente com tromboangeíte obliterante, continua sendo submetido à simpatectomia lombar e amputações progressivas de dedo, metatarso, pé e perna. A ausência de novas publicações nacionais a respeito da arterialização do arco venoso do pé demonstra que a cirurgia ainda não é praticada pelos cirurgiões brasileiros com a freqüência esperada.

\section{Referências}

1. Lengua Almora F, Herrera Zuloeta E, Kunlin J. Nuevos documentos experimentales de inversión circulatoria en miembro isquémico y de inyección retrógrada em piezas anatómicas. Diagnóstico (Peru). 1984;13:77-86.

2. Szilagyi D, Jay GD 3rd, Munnell ED. Femoral arteriovenous anastomosis in the treatment of occlusive disease. AMA Arch Surg. 1951;63:435-51.

3. Root HD, Cruz AB Jr. Effects of an arteriovenous fistula on the devascularized limb. JAMA. 1965;191:645-8.

4. Matolo NM, Cohen SE, Wolfmann EF Jr. Use of an arteriovenous fistula for treatment of the severely ischemic extremity: experimental evaluation. Ann Surg. 1976;5:622-5.

5. Courbier R, Jausseran JM, Reggi M. Sapheno-femoral shunt in severe ischaemia of the lower limbs. J Chir (Paris). 1973; 105:441-8. 
6. Pokrovskii AV, Dan VN, Khorovets AG, Chupin AV. [Arterialization of venous blood flow in the foot in the treatment of severe ischaemia in patients with crural arterial occlusions and non-functioning plantar arch]. Khirurgiia (Mosk). 1990;5:3542.

7. Lengua FA, Helfer LV. Técnica de arterialización de la red venosa del pie. Rev Sand Polic. 1974;35:203-10.

8. Lengua F, Nuss JM, Lechner R, Kunlin J. Arterialization of the venous network of the foot through a bypass in severe arteriophatic ischemia. J Cardiovasc Surg (Torino). 1984;25:357-60.

9. Lengua F, Nuss JM, Buffet JM, Lechner R. Etude comparative de deux modalités d'artérialisation des veines du pied en ischémie critique. J Chir (Paris). 1993;130:12-9.

10. Lengua $F$, Langeron P. Le pontage d'artérialisation veineuse distale peut-il être bénéfique au pied diabétique avec nécrose? Chirurgie. 1994-1995;120:143-52.

11. Lengua F, Cohen R, L'Huillier B, Buffet JM. Arteriovenous revascularization for lower limb salvage in unreconstructible arterial occlusive disease (long term outcome). Vasa. 1995;24:261-9.

12. Lengua F, La Madrid A, Acosta C, et al. L'artérialisation des veines du pied pour sauvetage de membre chez l'artéritique. Technique et résultats. Ann Chir. 2001;126:629-38.

13. Chen XS, Lin T, Chen DL, Guan YB. Venous arterialization in the treatment of extensive arterial occlusions of lower extremities. J Surg Concepts Pract. 1998;3:219-21.

14. Taylor RS, Belli AM, Jacob S. Distal venous arterialisation for salvage of critically ischaemic inoperable limbs. Lancet. 1999;354:1962-5.

15. Engelke C, Morgan RA, Quarmby JW, Taylor RS, Belli AM. Distal venous arterialization for lower limb salvage: angiographic appearances and interventional procedures. Radiographics. 2001;21:1239-48; discussion 1248-50.
16. Rowe VL, Hood DB, Liphan J, et al. Initial experience with dorsal venous arch arterialization for limb salvage. Ann Vasc Surg. 2002;16:187-92.

17. Ozbeck C, Kestelli M, Emrecan B, et al. A novel approach: ascending venous arterialization for atherosclerosis obliterans. Eur J Vasc Endovasc Surg. 2005;29:47-51

18. Busato CR, Utrabo CAL, Housome JK, Gomes RZ. Arterialização do arco venoso do pé para tratamento da isquemia crítica sem leito distal. Cir Vasc Angiol. 1999;15:117-21.

19. Gasparis AP, Noor S, Da Silva MS, Tassiopoulos AK, Semel L. Distal venous arterialization for limb salvage--a case report. Vasc Endovascular Surg. 2002;36:469-72

20. Kauffman P. Tromboangeíte obliterante. In: Maffei FHA, editor. Doenças vasculares periféricas. Rio de Janeiro: Medsi; 2002. vol.2, p.1271-9.

21. Lofgren EP, Myers TT, Lofgren KA, Kuster G. The venous valves of the foot and ankle. Surg Gynecol Obstet. 1968;127:289-90.

22. Garrido MB. Anatomia médico cirúrgica do sistema venoso dos membros inferiores. In: Maffei FHA. Doenças vasculares periféricas. $3^{\text {a }}$ ed. Rio de Janeiro: Medsi; 2002. vol.1, p. $133-67$.

23. Lu XW, Idu MM, Ubbink DT, Legemate DA. Meta-analysis of the clinical effectiveness of venous arterialization for salvage of critically ischaemic limbs. Eur J Vasc Endovasc Surg. 2006;31:493-9.

Correspondência:

César Roberto Busato

Rua Saldanha da Gama, 425

CEP 84015-130 - Ponta Grossa, PR

Tel.: (42) 3224.3288

E-mail: crbusato@brturbo.com.br 